\title{
Chlamydia pneumoniae-Mediated Inflammation in Atherosclerosis: A Meta-Analysis
}

\author{
Simone Filardo, ${ }^{1}$ Marisa Di Pietro, ${ }^{1}$ Alessio Farcomeni, ${ }^{2}$ \\ Giovanna Schiavoni, ${ }^{1}$ and Rosa Sessa ${ }^{1}$ \\ ${ }^{1}$ Section of Microbiology, Department of Public Health and Infectious Diseases, "Sapienza" University, Rome, Italy \\ ${ }^{2}$ Section of Statistic, Department of Public Health and Infectious Diseases, "Sapienza” University, Rome, Italy \\ Correspondence should be addressed to Rosa Sessa; rosa.sessa@uniromal.it
}

Received 8 May 2015; Accepted 15 July 2015

Academic Editor: Uma Nagarajan

Copyright (C) 2015 Simone Filardo et al. This is an open access article distributed under the Creative Commons Attribution License, which permits unrestricted use, distribution, and reproduction in any medium, provided the original work is properly cited.

\begin{abstract}
Several studies have attempted to relate the C. pneumoniae-mediated inflammatory state with atherosclerotic cardiovascular diseases, providing inconsistent results. Therefore, we performed a meta-analysis to clarify whether C. pneumoniae may contribute to the pathogenesis of atherosclerosis by enhancing inflammation. 12 case-control, 6 cross-sectional, and 7 prospective studies with a total of 10,176 patients have been included in this meta-analysis. Odds Ratio (OR) with a 95\% confidence interval was used to assess the seroprevalence of $C$. pneumoniae and differences between levels of inflammatory markers were assessed by standard mean differences. Publication bias was performed to ensure the statistical power. hsCRP, fibrinogen, interleukin- (IL-) 6 , TNF- $\alpha$, and IFN- $\gamma$ showed a significant increase in patients with atherosclerosis compared to healthy controls $(P<0.05)$, along with a higher seroprevalence of $C$. pneumoniae (OR of 3.11, 95\% CI: 2.88-3.36, $P<0.001$ ). More interestingly, hsCRP, IL-6, and fibrinogen levels were significantly higher in $C$. pneumoniae IgA seropositive compared to seronegative atherosclerotic patients $(P<0.0001)$. In conclusion, the present meta-analysis suggests that $C$. pneumoniae infection may contribute to atherosclerotic cardiovascular diseases by enhancing the inflammatory state, and, in particular, seropositivity to C. pneumoniae IgA, together with hsCRP, fibrinogen, and IL-6, may be predictive of atherosclerotic cardiovascular risk.
\end{abstract}

\section{Introduction}

Atherosclerosis, a chronic inflammatory disease of multifactorial aetiology, typically begins with endothelial dysfunction followed by low density lipoprotein (LDL) infiltration of the arterial intima, mononuclear cell recruitment into vascular wall, and the differentiation of macrophages in foam cells $[1,2]$. Macrophages and foam cells, within the evolving atherosclerotic lesion, secrete various proinflammatory cytokines, such as interleukin- (IL-) 6, interferon(IFN-) $\gamma$, tumor necrosis factor- (TNF-) $\alpha$, and chemokines, including intercellular adhesion molecule- (ICAM-) 1 and vascular cell adhesion molecule- (VCAM-) 1. In response to proinflammatory cytokines, endothelial cells express high levels of leukocyte adhesion molecules on their surface, leading to further mononuclear cell recruitment and, hence, to a chronic inflammatory state $[3,4]$. As a result, the formation, progression, and destabilisation of atherosclerotic plaque occur, leading to cardiovascular diseases, a major public health problem in developed countries, accounting for one-third of all deaths worldwide [5].

In the last decades, several infectious agents have been related to the pathogenesis of atherosclerotic cardiovascular diseases, and current opinion is that the most implicated pathogen is Chlamydia pneumoniae, an obligate intracellular microorganism, known as being responsible for respiratory tract infections $[6,7]$.

The association between C. pneumoniae and atherosclerotic cardiovascular diseases has been well documented by seroepidemiological studies [8-12], direct detection of microorganism within atherosclerotic plaque [13-17], and in vivo studies showing an atherosclerotic lesion exacerbation following C. pneumoniae inoculation of hyperlipidemic animal models [18-23]. 
C. pneumoniae is presumed to play a role in atherosclerotic cardiovascular diseases for its ability to systematically disseminate from the lung through peripheral blood mononuclear cells and to localise in extrapulmonary tissues, such as the vascular wall [24-28]. Once being inside the vascular tissue, $C$. pneumoniae has been shown to act directly on the cells involved in atherosclerotic process, contributing to endothelial dysfunction, foam cell formation, vascular smooth muscle cell (VSMC) proliferation and migration, and platelet aggregation [29-33]. Indeed, C. pneumoniae is able to multiply within macrophages, platelets, endothelial cells, and VSMCs and to induce the elicitation of proinflammatory cytokines, such as IL- 6 , IFN- $\gamma$, and TNF- $\alpha$, and adhesion molecules, such as ICAM-1 and VCAM-1, as well as reactive oxygen species, thus contributing to the chronic inflammatory state responsible for the initiation, progression, and destabilisation of atherosclerotic plaque [34-40].

Specifically, C. pneumoniae persistent form seems to be responsible for chronic infection and, hence, for the inflammatory process underlying atherosclerosis, since it is able to endure for a long time inside host cells [41, 42].

In addition to the direct effect on vascular cells, previously described, C. pneumoniae has also shown to contribute to the systemic inflammation involved in the pathogenesis of atherosclerotic cardiovascular diseases, as evidenced by high levels of IL- 6 and high sensitivity c-reactive protein (hsCRP) $[7,43]$.

Given the central role of inflammation in the atherosclerotic process and the inflammatory effects of $C$. pneumoniae, several studies have attempted to relate the C. pneumoniaemediated inflammatory state with atherosclerotic cardiovascular diseases, providing inconsistent results.

We performed, therefore, a meta-analysis to clarify whether C. pneumoniae may contribute to the pathogenesis of atherosclerotic cardiovascular diseases by the means of enhanced inflammatory state.

\section{Materials and Methods}

2.1. Search Strategy. We performed a systematic search of all articles in journals indexed on the electronic databases PubMed and Scopus up to December 2014. The search terms used were "chlamydia pneumoniae" or "chlamydophila pneumoniae," "inflammatory markers" or "inflammation," "atherosclerosis," and "cardiovascular disease." The reference lists of reviews and retrieved articles were hand-searched simultaneously. When more than one of the same patient population was included in several publications, only the most recent or complete study was included in this metaanalysis.

2.2. Inclusion and Exclusion Criteria. The inclusion criteria in this meta-analysis were as follows: (i) studies comparing atherosclerotic patients with healthy subjects (control group) in relation to the seropositivity to C. pneumoniae IgG or IgA, (ii) studies comparing atherosclerotic patients in relation to the seropositivity to C. pneumoniae IgG or IgA, and (iii) studies analysing the levels of inflammatory markers.
Case reports, reviews, letters, and studies which did not present their results in a correct and/or explicit manner, animal studies, and studies where the control groups were made up of patients with other chronic inflammatory disorders were excluded from our meta-analysis. We did not define any minimum number of patients to include a study in our metaanalysis.

2.3. Data Extraction. Information was carefully extracted from all eligible publications independently by two authors according to the inclusion and exclusion criteria listed above. Disagreement was resolved by discussion between the two authors. The following data were collected from each study: first author's surname, year of publication, study design, type of cases, type of controls, total numbers of cases and controls, prevalence of traditional risk factors for CVD (obesity, diabetes, smoking, dyslipidemia, and hypertension), the prevalence of C. pneumoniae antibodies (IgA or IgG), determined by microimmunofluorescence or ELISA, and the levels of inflammatory markers (CRP, IL-6, fibrinogen, IFN$\gamma$, TNF- $\alpha$, ICAM-1, and VCAM-1).

Only inflammatory markers analysed by at least two studies were included in our meta-analysis.

2.4. Statistical Analysis. Differences between groups were assessed by means of differences in averages and standard deviations of the difference, as markers assessed were all continuous. Standard errors were computed after a normal approximation. Pooled effects were computed by means of a meta-analysis. The meta-analysis conducted was strictly under heterogeneity among studies, by means of a hierarchical Bayesian model. Effect sizes were assumed to be normally distributed. Each study effect was assumed to arise from a Gaussian centered on a study-specific effect and the extracted standard error, inflated by $25 \%$ to obtain a conservative statement. The study-specific effect was assumed to be Gaussian, centered on an unknown pooled effect, which is the main object of interest. An informative prior was used for the variance of the pooled effect, as an inverse Gamma centered on an estimator obtained with a moment-based approach (inflated by $25 \%$ to obtain a conservative statement). We also estimated posterior probabilities of no difference $(P) . P$ of less than 0.05 was considered as statistically significant. Potential publication bias was estimated using Egger's linear regression test and funnel plots. Sensitivity analyses were assessed by deleting each study; in all cases, pooled estimates were very stable.

All data were collected using Microsoft Office Excel 2007; statistical analyses were performed using R software.

\section{Results}

3.1. Study Characteristics. 25 studies concerning the association of $C$. pneumoniae and atherosclerosis through inflammation met the inclusion criteria and comprised 12 casecontrol, 6 cross-sectional, and 7 prospective studies [4468]. Overall, a total of 3633 cases and 2781 controls for the case-control analysis, 1593 seropositive and 915 seronegative 
TABLE 1: Characteristics of the studies included in the case-control analysis.

\begin{tabular}{|c|c|c|c|c|c|c|c|}
\hline First author & Year & Country & Cases & Number of cases & Number of controls & Study design & Serology assay \\
\hline Mundkur [68] & 2012 & India & CAD & 433 & 433 & Prospective & ELISA \\
\hline Haider [67] & 2011 & India & CAD & 63 & 40 & Cross-sectional & ELISA \\
\hline Jha [65] & 2009 & India & CAD & 192 & 192 & Case-control & ELISA \\
\hline Jafarzadeh [64] & 2008 & Iran & IHD & 62 & 31 & Case-control & ELISA \\
\hline Videm [63] & 2007 & Norway & CAD & 131 & 103 & Case-control & ELISA \\
\hline Corrado [59] & 2006 & Italy & A & 456 & 212 & Prospective & ELISA \\
\hline Kaperonis [61] & 2006 & Greece & PAD & 51 & 30 & Cross-sectional & ELISA \\
\hline Romano Carratelli [62] & 2006 & Italy & CAD & 60 & 20 & Case-control & MIF \\
\hline Adiloglu [57] & 2005 & Turkey & A & 88 & 91 & Case-control & ELISA \\
\hline Lanza $[55]$ & 2004 & Italy & SA, cardiac SX & 104 & 60 & Case-control & MIF \\
\hline Linares-Palomino [56] & 2004 & Spain & PAD & 64 & 50 & Case-control & MIF \\
\hline Biasucci $[51]$ & 2003 & Italy & $\mathrm{UA}, \mathrm{MI}$ & 259 & 100 & Prospective & MIF \\
\hline Georges [53] & 2003 & Germany & SA, UA & 991 & 333 & Case-control & MIF \\
\hline Bloemenkamp [49] & 2002 & Netherlands & PAD & 228 & 643 & Case-control & ELISA \\
\hline Gattone [46] & 2001 & Italy & MI & 120 & 120 & Case-control & MIF \\
\hline Anderson [44] & 1998 & USA & $\mathrm{CAD}, \mathrm{MI}$ & 331 & 323 & Case-control & MIF \\
\hline
\end{tabular}

CAD, coronary artery disease; IHD, ischemic heart disease; A, atherosclerosis; PAD, peripheral artery disease; SA, stable angina; SX, syndrome X; UA, unstable angina; MI, myocardial infarction.

TABLE 2: Characteristics of the studies included in the C. pneumoniae IgG and IgA analysis.

\begin{tabular}{|c|c|c|c|c|c|c|c|}
\hline First author & Year & Country & Cases & Number of cases & Number of controls & Study design & Serology assay \\
\hline \multicolumn{8}{|c|}{$\operatorname{IgG}$} \\
\hline Nazmi [66] & 2010 & USA & CAD & 697 & 288 & Cross-sectional & MIF \\
\hline Jitsuiki [60] & 2006 & Japan & A & 136 & 123 & Cross-sectional & ELISA \\
\hline Adiloglu [57] & 2005 & Turkey & A & 227 & 17 & Case-control & ELISA \\
\hline Zairis [54] & 2003 & Greece & SA, UA & 182 & 214 & Prospective & MIF \\
\hline Altman [48] & 2002 & Argentina & CAD & 107 & 52 & Case-control & MIF \\
\hline Schumacher [50] & 2002 & Norway & CAD & 119 & 74 & Cross-sectional & ELISA \\
\hline Sander [47] & 2001 & Germany & TIA, IS & 125 & 147 & Prospective & MIF \\
\hline \multicolumn{8}{|c|}{$\operatorname{Ig} \mathrm{A}$} \\
\hline Jha [65] & 2009 & India & CAD & 155 & 37 & Case-control & ELISA \\
\hline Jitsuiki [60] & 2006 & Japan & A & 92 & 167 & Cross-sectional & ELISA \\
\hline Schumacher [58] & 2005 & Norway & CAD & 63 & 130 & Cross-sectional & MIF \\
\hline Gabriel [52] & 2003 & Sweden & CAD & 38 & 15 & Prospective & MIF \\
\hline Zairis [54] & 2003 & Greece & SA, UA & 87 & 214 & Prospective & MIF \\
\hline Toss [45] & 1998 & Sweden & UA & 93 & 163 & Prospective & MIF \\
\hline
\end{tabular}

CAD, coronary artery disease; A, atherosclerosis; SA, stable angina; UA, unstable angina; TIA, transient ischemic attack; IS, ischemic stroke.

patients for the C. pneumoniae IgG seropositive-seronegative analysis, and 528 seropositive and 726 seronegative patients for the C. pneumoniae IgA seropositive-seronegative analysis were considered. One publication had apparently overlapping cases with a second study, so that we extracted only the most relevant data from both studies. Table 1 presents the main characteristics of the included studies for the case-control analysis, whereas Table 2 presents the main characteristics of the included studies for the seropositive to seronegative analysis according to C. pneumoniae IgG and IgA. Controls were mainly healthy population and matched for age and sex.
3.2. Meta-Analysis Results. The meta-analysis was performed on 16 studies (case-controls analysis, Table 1) comparing atherosclerotic patients with healthy controls in relation to the seropositivity to $C$. pneumoniae and levels of inflammatory markers (hsCRP, IL-6, fibrinogen, TNF- $\alpha$, IFN- $\gamma$, ICAM-1, and VCAM-1) [44, 46, 49, 51, 53, 55-57, 59, 6165, 67, 68]. C. pneumoniae seroprevalence was 59.2\% (95\% CI: $57.8-60.7)$ in cases and $31.2 \%$ (95\% CI: $30.0-32.5)$ in controls, with an OR of 3.11 (95\% CI: 2.88-3.36, $P<$ 0.001). Concerning the inflammatory markers, hsCRP, IL-6, fibrinogen, TNF- $\alpha$, and IFN- $\gamma$ showed a significant increase 
TABLE 3: Summary of SMDs and 95\% CI of inflammatory marker levels in case-control analysis.

\begin{tabular}{lccccccc}
\hline & $\begin{array}{c}\text { hsCRP } \\
(\mathrm{mg} / \mathrm{L})\end{array}$ & $\begin{array}{c}\text { IL-6 } \\
(\mathrm{ng} / \mathrm{mL})\end{array}$ & $\begin{array}{c}\text { Fibrinogen } \\
(\mathrm{mg} / \mathrm{dL})\end{array}$ & $\begin{array}{c}\text { TNF- } \alpha \\
(\mathrm{pg} / \mathrm{mL})\end{array}$ & $\begin{array}{c}\text { IFN- } \gamma \\
(\mathrm{pg} / \mathrm{mL})\end{array}$ & $\begin{array}{c}\text { ICAM-1 } \\
(\mathrm{ng} / \mathrm{mL})\end{array}$ & $\begin{array}{c}\text { VCAM-1 } \\
(\mathrm{ng} / \mathrm{mL})\end{array}$ \\
\hline SMD & 6.360 & 7.861 & 5.627 & 9.467 & 4.320 & 0.001 & 3.722 \\
$95 \%$ CI & $5.76-6.96$ & $7.40-8.32$ & $0.65-10.60$ & $6.45-12.49$ & $2.32-6.32$ & $-0.25-0.25$ \\
$P$ value & $<0.0001$ & $<0.0001$ & 0.00895 & $<0.0001$ & $<0.0001$ & 0.49628 & 0.06611 \\
\hline
\end{tabular}

SMD, standard mean difference; CI, confidence interval.

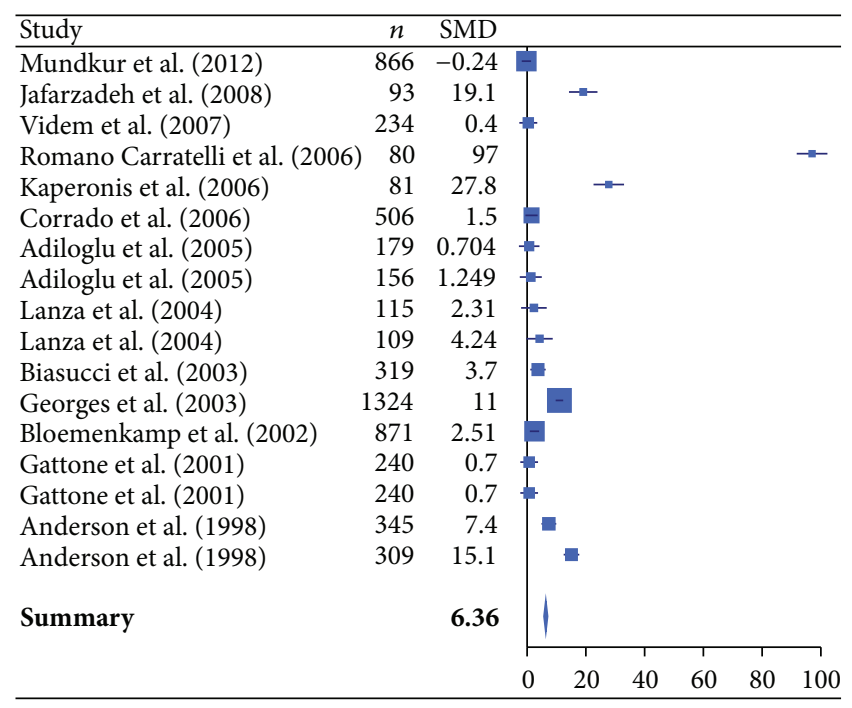

(a)

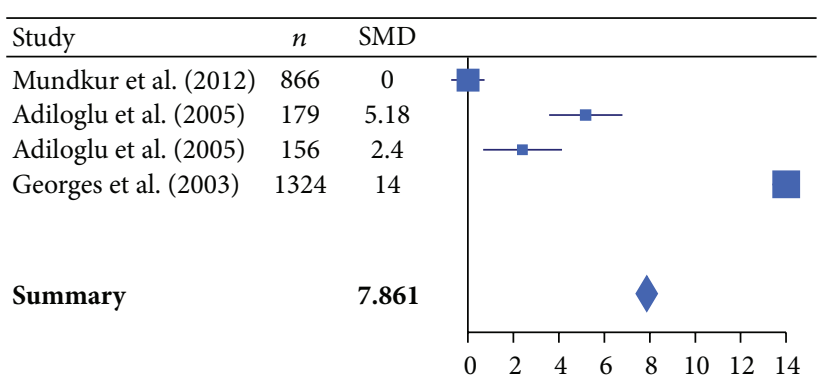

(b)

\begin{tabular}{|c|c|c|c|}
\hline Study & $n$ & SMD & \\
\hline Mundkur et al. (2012) & 866 & 272.1 & $-1-$ \\
\hline Romano Carratelli et al. (2006) & 80 & 300 & \\
\hline Kaperonis et al. (2006) & 81 & 2.46 & \\
\hline Corrado et al. (2006) & 506 & 50 & $=$ \\
\hline Linares-Palomino et al. (2004) & 114 & 12.69 & \\
\hline Georges et al. (2003) & 1324 & 9.1 & \\
\hline Gattone et al. (2001) & 240 & 28 & \\
\hline Gattone et al. (2001) & 240 & 28 & \\
\hline \multirow[t]{2}{*}{ Summary } & & 5.627 & \\
\hline & & $-400-200$ & $200 \quad 400 \quad 600$ \\
\hline
\end{tabular}

(c)

FIGURE 1: Forest plot of standardized mean differences (SMDs) of individual studies and pooled SMDs for hsCRP (a), IL-6 (b), and fibrinogen (c) in patients with atherosclerotic cardiovascular diseases and healthy controls.

in patients with atherosclerosis compared to health controls $(P<0.05)$, whereas the adhesion molecules ICAM-1 and VCAM-1 did not show any significant difference $(P>0.05)$ (Table 3, Figure 1).

In addition, the meta-analysis was also performed on studies comparing atherosclerotic patients seropositive to C. pneumoniae IgG or IgA with seronegative patients. Specifically, 7 studies comparing atherosclerotic patients seropositive to $C$. pneumoniae IgG with seronegative patients (C. pneumoniae IgG analysis, Table 2) [47, 48, 50, 54, 57, $60,66]$ and 6 studies comparing atherosclerotic patients seropositive to C. pneumoniae IgA with seronegative patients
(C. pneumoniae IgA analysis, Table 2) [45, 52, 54, 58, 60, 65], both according to levels of inflammatory markers (hsCRP, IL6 , fibrinogen, and IFN- $\gamma$ ), were evaluated.

For the C. pneumoniae IgG analysis, hsCRP, IL-6, and fibrinogen levels were significantly higher in seropositive compared to seronegative patients $(P<0.0001)$ (Figure 2).

For the C. pneumoniae IgA analysis, hsCRP, IL-6, and fibrinogen were markedly increased in seropositive patients $(P<0.01)$, whereas IFN- $\gamma$ did not show any significant difference between seropositive and seronegative patients $(P>0.05)$ (Figure 3). 


\begin{tabular}{|c|c|c|c|}
\hline Study & $n$ & SMD & \\
\hline Nazmi et al. (2010) & 985 & 0.012 & \\
\hline Jitsuiki et al. (2006) & 259 & 0.007 & 7 \\
\hline Adiloglu et al. (2005) & 244 & 0.0847 & $\longrightarrow$ \\
\hline Zairis et al. (2003) & 396 & 0.01 & \\
\hline Schumacher et al. (2002) & 193 & 0.131 & $\longrightarrow$ \\
\hline Altman et al. (2002) & 159 & $-0.1 \longrightarrow$ & \\
\hline Sander et al. (2001) & 272 & 0.1 & $\longrightarrow$ \\
\hline \multirow[t]{2}{*}{ Summary } & & 0.029 & \\
\hline & & $-0.1-0.05$ & \begin{tabular}{ll|l|l} 
& & & \\
0 & 0.05 & 0.1 & 0.15
\end{tabular} \\
\hline
\end{tabular}

(a)

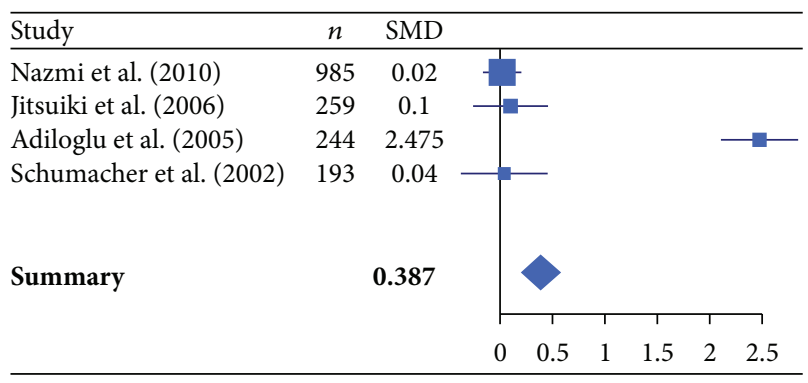

(b)

\begin{tabular}{|c|c|c|c|c|c|c|c|}
\hline Study & $n$ & SMD & & & & & \\
\hline Nazmi et al. (2010) & 985 & -2 & 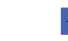 & - & & & \\
\hline Schumacher et al. (2002) & 193 & 45 & & & & & - \\
\hline Altman et al. (2002) & 159 & -10 & $\rightarrow-$ & & & & \\
\hline \multirow[t]{2}{*}{ Summary } & & 3.81 & & $\uparrow$ & & & \\
\hline & & & -10 & $0 \quad 10$ & 20 & 30 & 40 \\
\hline
\end{tabular}

(c)

FIGURE 2: Forest plot of standardized mean differences (SMDs) of individual studies and pooled SMDs for hsCRP (a), IL-6 (b), and fibrinogen (c) in C. pneumoniae IgG seropositive and seronegative patients with atherosclerotic cardiovascular diseases.

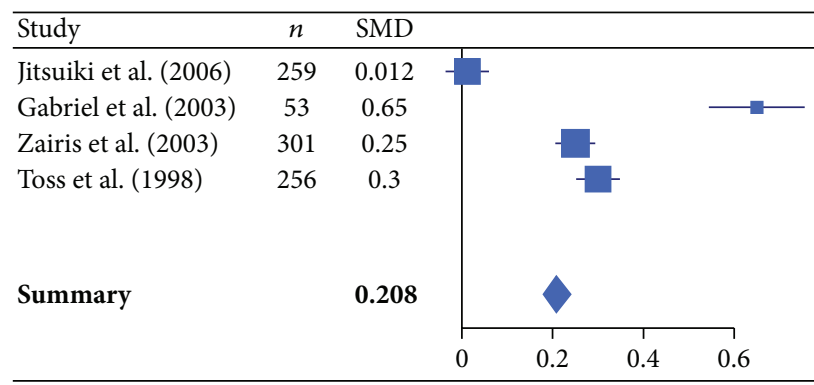

(a)

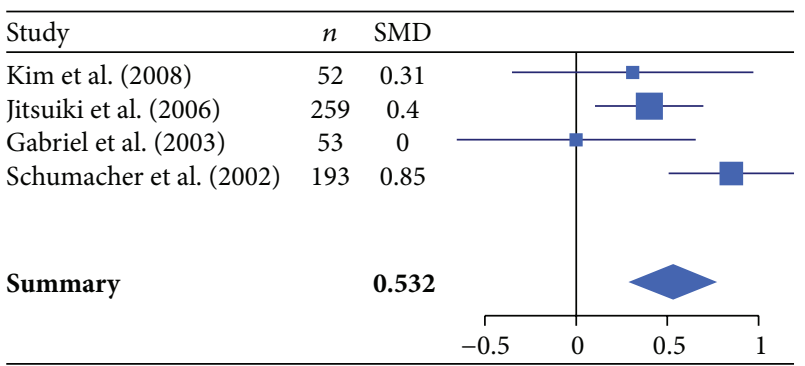

(b)

\begin{tabular}{lccccccc}
\hline Study & $n$ & SMD & & & & \\
\hline Gabriel et al. (2003) & 53 & 60 & & & & \\
Schumacher et al. (2002) & 193 & 30 & - & & & \\
Toss et al. (1998) & 256 & 24 & - & & & & \\
& & & & & & & \\
Summary & & 29.67 & & & & & \\
& & & & 30 & 40 & 50 & 60 \\
\end{tabular}

(c)

FIGURE 3: Forest plot of standardized mean differences (SMDs) of individual studies and pooled SMDs for hsCRP (a), IL-6 (b), and fibrinogen (c) in C. pneumoniae IgA seropositive and seronegative patients with atherosclerotic cardiovascular diseases.

Results are independent of the main confounding factors as the meta-analysis was computed under heterogeneity. We also have verified that they could not form a relationship with mean age and study proportions of males, patients with diabetes, hypertension, obesity, smoking, dyslipidemia, and risk factors for CVDs.
3.3. Publication Bias. Funnel plots and Egger's test were performed to assess publication bias. Publication bias was detected only for meta-analysis of fibrinogen with $\operatorname{IgA}(P=$ $0.012)$. In particular no publication bias was detected for hsCRP for $\operatorname{IgA}(P=0.200), \operatorname{IgG}(P=0.890)$, and case-control data $(P=0.122)$. 


\section{Discussion}

Atherosclerosis, precursor to cardiovascular diseases, is recognised as a chronic inflammatory disease of large arteries, involving cytokines, such as IFN- $\gamma$ and TNF- $\alpha$, adhesion molecules, such as ICAM-1 and VCAM-1, and several plasma inflammatory markers, such as hsCRP, IL-6, and fibrinogen $[2,3]$. The latter have been demonstrated to better correlate with the chronic inflammation underlying atherosclerotic cardiovascular diseases; in fact, IL-6, a proinflammatory cytokine that plays a role in the instability of a vulnerable plaque, and hsCRP, an acute-phase protein, appear to be strong independent predictors of cardiovascular events [3]. Lastly, fibrinogen, a substrate leading to the generation of thrombin, the end point of the hemostatic process, is involved in the early formation and growth of the atherosclerotic plaque [69].

As previously described, C. pneumoniae has been suggested to contribute to the chronic inflammation underlying the atherosclerotic process, as evidenced by elevated levels of circulating inflammatory markers in atherosclerotic patients. Gattone et al., in 2001, have suggested, for the first time, the association of C. pneumoniae seropositivity and high hsCRP levels, with increased risk of myocardial infarction [46]. Since then, other studies $[47,49,50,52,57,61,64,67]$ have supported the relationship between $C$. pneumoniae infection and atherogenesis through systemic inflammation. On the other hand, some studies have failed to demonstrate such association $[44,48,55,68]$, thus making it difficult to draw conclusions.

To the best of our knowledge, our study is the first metaanalysis to evaluate whether C. pneumoniae may contribute to the pathogenesis of atherosclerotic cardiovascular diseases by the means of enhanced inflammation, assessed by levels of inflammatory markers.

The first result of our meta-analysis showed a significant increase in hsCRP, fibrinogen, IL-6, TNF- $\alpha$, and IFN- $\gamma$ in atherosclerotic patients compared to healthy controls. These data, together with the higher seroprevalence of $C$. pneumoniae in atherosclerotic patients compared to healthy controls $(P<0.001)$, suggest that $C$. pneumoniae infection may contribute to the chronic inflammation underlying the development and progression of atherosclerosis, even though it is not possible to establish a causal relationship.

Consequently, in order to better define the contribution of C. pneumoniae on the inflammation underlying atherosclerosis, we performed a further analysis on studies comparing the levels of inflammatory markers in atherosclerotic patients seropositive to C. pneumoniae IgG or IgA with seronegative patients.

The more relevant data is that C. pneumoniae IgA seropositivity was overall more strongly related to the inflammatory state than IgG seropositivity. Indeed, our results showed an increase of inflammatory marker levels, such as hsCRP, fibrinogen, and IL-6, in IgA seropositive patients. Specifically, the standard mean differences of hsCRP, fibrinogen, and IL-6 were, respectively, 7.17, 7.79, and 1.37 times higher in the IgA compared to the IgG analysis, thus showing that seropositivity to C. pneumoniae IgA might better correlate to a chronic inflammatory state in patients with atherosclerosis. This is not particularly surprising since $C$. pneumoniae IgA is considered a marker of chronic infection, known to be involved in chronic disorders.

The main strength of our meta-analysis is the statistical robustness of our data, since the results were not affected by exclusion of any specific study from the pooled analysis.

However, our meta-analysis has potential limitations. First, since multiple infectious agents, labeled as infectious burden, rather than any single pathogen, have been shown to contribute to the pathogenesis of atherosclerosis, the inflammatory effect of $C$. pneumoniae might be overestimated. Of note, in all the studies included in our metaanalysis, data regarding other infectious agents were missing or incomplete and, hence, we were not able to quantify their influence on chronic inflammation. Second, potential bias might be introduced in that not all the included studies reported data concerning the confounding factors. Lastly, two different diagnostic tests, along with different cutoffs, were used for detecting $C$. pneumoniae seropositivity in the included studies and, hence, may partly account for further bias.

\section{Conclusions}

In conclusion, despite some limitations, our study suggests that C. pneumoniae infection may contribute to atherosclerotic cardiovascular diseases by enhancing the inflammatory state, as demonstrated by increased levels of systemic inflammatory markers. Furthermore, seropositivity to $C$. pneumoniae IgA, together with hsCRP, fibrinogen, and IL-6, may be predictive of atherosclerotic cardiovascular risk.

In the future, large scale, prospective, and well-designed studies should be needed to deepen our knowledge concerning the causative role of C. pneumoniae in inflammation in atherosclerotic cardiovascular diseases.

\section{Conflict of Interests}

The authors declare that they have no conflict of interests.

\section{Acknowledgment}

This study was supported by grants from Center for Social Disease Research, "Sapienza" University Rome, to Rosa Sessa.

\section{References}

[1] M. Rafieian-Kopaei, M. Setorki, M. Doudi, A. Baradaran, and H. Nasri, "Atherosclerosis: process, indicators, risk factors and new hopes," International Journal of Preventive Medicine, vol. 5, no. 8, pp. 927-946, 2014.

[2] H. Mangge, K. Becker, D. Fuchs et al., "Antioxidants, inflammation and cardiovascular disease," World Journal of Cardiology, vol. 6, no. 6, pp. 462-477, 2014.

[3] L. Stoner, A. A. Lucero, B. R. Palmer, L. M. Jones, J. M. Young, and J. Faulkner, "Inflammatory biomarkers for predicting cardiovascular disease," Clinical Biochemistry, vol. 46, no. 15, pp. 1353-1371, 2013. 
[4] S. Pant, A. Deshmukh, G. S. Gurumurthy et al., "Inflammation and atherosclerosis-revisited," Journal of Cardiovascular Pharmacology and Therapeutics, vol. 19, no. 2, pp. 170-178, 2014.

[5] World Health Organization, Global Status Report on Noncommunicable Diseases 2010, World Health Organization, Geneva, Switzerland, 2011.

[6] M. E. Rosenfeld and L. A. Campbell, "Pathogens and atherosclerosis: update on the potential contribution of multiple infectious organisms to the pathogenesis of atherosclerosis," Thrombosis and Haemostasis, vol. 106, no. 5, pp. 858-867, 2011.

[7] R. Sessa, M. Di Pietro, S. Filardo, and O. Turriziani, "Infectious burden and atherosclerosis: a clinical issue," World Journal of Clinical Cases, vol. 2, no. 7, pp. 240-249, 2014.

[8] P. Saikku, M. Leinonen, K. Mattila et al., "Serological evidence of an association of a novel Chlamydia, TWAR, with chronic coronary heart disease and acute myocardial infarction," The Lancet, vol. 332, no. 8618, pp. 983-986, 1988.

[9] J. Boman and M. R. Hammerschlag, "Chlamydia pneumoniae and atherosclerosis: critical assessment of diagnostic methods and relevance to treatment studies," Clinical Microbiology Reviews, vol. 15, no. 1, pp. 1-20, 2002.

[10] H. C. Jha, P. Srivastava, R. Sarkar, J. Prasad, and A. Mittal, "Chlamydia pneumoniae IgA and elevated level of IL-6 may synergize to accelerate coronary artery disease," Journal of Cardiology, vol. 52, no. 2, pp. 140-145, 2008.

[11] R. Joshi, B. Khandelwal, D. Joshi, and O. P. Gua, "Chlamydophila pneumoniae infection and cardiovascular disease," North American Journal of Medical Sciences, vol. 5, no. 3, pp. 169-181, 2013.

[12] N. Sakurai-Komada, H. Iso, K. A. Koike et al., "Association between Chlamydophila pneumoniae infection and risk of coronary heart disease for Japanese: the JPHC study," Atherosclerosis, vol. 233, no. 2, pp. 338-342, 2014.

[13] A. Shor and J. I. Phillips, "Histological and ultrastructural findings suggesting an initiating role for Chlamydia pneumoniae in the pathogenesis of atherosclerosis: a study of 50 cases," Cardiovascular Journal of Southern Africa, vol. 11, no. 1, pp. 1623, 2000.

[14] D. Virok, Z. Kis, L. Kari et al., "Chlamydophila pneumoniae and human cytomegalovirus in atherosclerotic carotid plaquescombined presence and possible interactions," Acta Microbiologica et Immunologica Hungarica, vol. 53, no. 1, pp. 35-50, 2006.

[15] H. C. Jha, P. Srivastava, A. Divya, J. Prasad, and A. Mittal, "Prevalence of Chlamydophila pneumoniae is higher in aorta and coronary artery than in carotid artery of coronary artery disease patients," APMIS, vol. 117, no. 12, pp. 905-911, 2009.

[16] B. Atik, S. C. Johnston, and D. Dean, "Association of Carotid Plaque Lp-PLA2 with Macrophages and Chlamydia pneumoniae infection among patients at risk for stroke," PLoS ONE, vol. 5, no. 6, Article ID el1026, 2010.

[17] A. Luque, M. M. Turu, N. Rovira, J. O. Juan-Babot, M. Slevin, and J. Krupinski, "Early atherosclerotic plaques show evidence of infection by Chlamydia pneumoniae," Frontiers in Bioscience, vol. 4, no. 7, pp. 2423-2432, 2012.

[18] J. B. Muhlestein, "Chlamydia pneumoniae-induced atherosclerosis in a rabbit model,” Journal of Infectious Diseases, vol. 181, supplement 3, pp. S505-S507, 2000.

[19] E. Blessing, L. A. Campbell, M. E. Rosenfeld, N. Chough, and C.-C. Kuo, "Chlamydia pneumoniae infection accelerates hyperlipidemia induced atherosclerotic lesion development in C57BL/6J mice," Atherosclerosis, vol. 158, no. 1, pp. 13-17, 2001.
[20] S. Chen, K. Shimada, W. Zhang, G. Huang, T. R. Crother, and M. Arditi, "IL-17A is proatherogenic in high-fat diet-induced and Chlamydia pneumoniae infection-accelerated atherosclerosis in mice," Journal of Immunology, vol. 185, no. 9, pp. 5619-5627, 2010.

[21] F.-Y. Lin, Y.-W. Lin, C.-Y. Huang et al., "GroEL1, a heat shock protein 60 of Chlamydia pneumoniae, induces lectinlike oxidized low-density lipoprotein receptor 1 expression in endothelial cells and enhances atherogenesis in hypercholesterolemic rabbits," The Journal of Immunology, vol. 186, no. 7, pp. 4405-4414, 2011.

[22] C.-Y. Huang, C.-M. Shih, N.-W. Tsao et al., "GroEL1, from Chlamydia pneumoniae, induces vascular adhesion molecule 1 expression by p37AUF1 in endothelial cells and hypercholesterolemic rabbit," PLoS ONE, vol. 7, no. 8, Article ID e42808, 2012.

[23] R. Sorrentino, A. Yilmaz, K. Schubert et al., "A single infection with Chlamydia pneumoniae is sufficient to exacerbate atherosclerosis in ApoE deficient mice," Cellular Immunology, vol. 294, no. 1, pp. 25-32, 2015.

[24] S. S. Wang, M. L. C. Tondella, A. Bajpai et al., "Circulating Chlamydia pneumoniae DNA and advanced coronary artery disease," International Journal of Cardiology, vol. 118, no. 2, pp. 215-219, 2007.

[25] M. Edvinsson, E. Hjelm, S. Thelin, G. Friman, and C. NyströmRosander, "Presence of Chlamydophila pneumoniae DNA but not mRNA in stenotic aortic heart valves," International Journal of Cardiology, vol. 143, no. 1, pp. 57-62, 2010.

[26] L. Mannonen, E. Markkula, and M. Puolakkainen, "Analysis of Chlamydia pneumoniae infection in mononuclear cells by reverse transcription-PCR targeted to chlamydial gene transcripts," Medical Microbiology and Immunology, vol. 200, no. 3, pp. 143-154, 2011.

[27] M. Di Pietro, G. Schiavoni, V. Sessa, F. Pallotta, G. Costanzo, and R. Sessa, "Chlamydia pneumoniae and osteoporosis-associated bone loss: a new risk factor?” Osteoporosis International, vol. 24, no. 5, pp. 1677-1682, 2013.

[28] M. Di Pietro, S. Filardo, S. Cazzavillan et al., "Could past Chlamydial vascular infection promote the dissemination of Chlamydia pneumoniae to the brain?" Journal of Biological Regulator and Homeostatic Agents, vol. 27, no. 1, pp. 155-164, 2013.

[29] H. Kälvegren, J. Andersson, M. Grenegård, and T. Bengtsson, "Platelet activation triggered by Chlamydia pneumoniae is antagonized by 12-lipoxygenase inhibitors but not cyclooxygenase inhibitors," European Journal of Pharmacology, vol. 566, no. 1-3, pp. 20-27, 2007.

[30] J. M. Kern, V. Maass, and M. Maass, “Chlamydia pneumoniae adversely modulates vascular cell properties by direct interaction with signalling cascades," Thrombosis and Haemostasis, vol. 102, no. 6, pp. 1064-1070, 2009.

[31] M. N. Chahine, J. Deniset, E. Dibrov et al., "Oxidized LDL promotes the mitogenic actions of Chlamydia pneumoniae in vascular smooth muscle cells," Cardiovascular Research, vol. 92, no. 3, pp. 476-483, 2011.

[32] L. A. Campbell, A. W. Lee, M. E. Rosenfeld, and C.-C. Kuo, "Chlamydia pneumoniae induces expression of pro-atherogenic factors through activation of the lectin-like oxidized LDL receptor-1," Pathogens and Disease, vol. 69, no. 1, pp. 1-6, 2013.

[33] G.-J. Zhao, Z.-C. Mo, S.-L. Tang et al., "Chlamydia pneumoniae negatively regulates ABCA1 expression via TLR2-Nuclear 
factor-kappa B and miR-33 pathways in THP-1 macrophagederived foam cells," Atherosclerosis, vol. 235, no. 2, pp. 519-525, 2015.

[34] C. A. Gaydos, "Growth in vascular cells and cytokine production by Chlamydia pneumoniae," Journal of Infectious Diseases, vol. 181, no. 6, pp. S473-S478, 2000.

[35] F. Cao, A. Castrillo, P. Tontonoz, F. Re, and G. I. Byrne, “Chlamydia pneumoniae-induced macrophage foam cell formation is mediated by toll-like receptor 2," Infection and Immunity, vol. 75, no. 2, pp. 753-759, 2007.

[36] M. Högdahl, G. Söderlund, and E. Kihlström, "Expression of chemokines and adhesion molecules in human coronary artery endothelial cells infected with Chlamydia (Chlamydophila) pneumoniae," APMIS, vol. 116, no. 12, pp. 1082-1088, 2008.

[37] W. Liu, P. He, B. Cheng, C.-L. Mei, Y.-F. Wang, and J.-J. Wan, "Chlamydia pneumoniae disturbs cholesterol homeostasis in human THP-1 macrophages via JNK-PPAR $\gamma$ dependent signal transduction pathways," Microbes and Infection, vol. 12, no. 1415, pp. 1226-1235, 2010.

[38] M. Di Pietro, F. de Santis, G. Schiavoni, S. Filardo, and R. Sessa, "Resveratrol in Chlamydia pneumoniae-induced foam cell formation and interleukin-17A synthesis," Journal of Biological Regulators and Homeostatic Agents, vol. 27, no. 2, pp. 509518, 2013.

[39] B. Wang, L. Zhang, T. Zhang et al., "Chlamydia pneumoniae infection promotes vascular smooth muscle cell migration through a toll-like receptor 2-related signaling pathway," Infection and Immunity, vol. 81, no. 12, pp. 4583-4591, 2013.

[40] M. Di Pietro, S. Filardo, F. De Santis, P. Mastromarino, and R. Sessa, "Chlamydia pneumoniae and oxidative stress in cardiovascular disease: state of the art and prevention strategies," International Journal of Molecular Sciences, vol. 16, no. 1, pp. 724-735, 2015.

[41] R. V. Schoborg, "Chlamydia persistence-a tool to dissect Chlamydia-host interactions," Microbes and Infection, vol. 13, no. 7, pp. 649-662, 2011.

[42] M. Di Pietro, S. Filardo, F. De Santis, and R. Sessa, "New insights into Chlamydiae persistence: an energy metabolism strategy?" International Journal of Immunopathology and Pharmacology, vol. 26, no. 2, pp. 525-528, 2013.

[43] L. A. Campbell, K. Yaraei, B. Van Lenten et al., "The acute phase reactant response to respiratory infection with Chlamydia pneumoniae: implications for the pathogenesis of atherosclerosis," Microbes and Infection, vol. 12, no. 8-9, pp. 598-606, 2010.

[44] J. L. Anderson, J. F. Carlquist, J. B. Muhlestein, B. D. Horne, and S. P. Elmer, "Evaluation of c-reactive protein, an inflammatory marker, and infectious serology as risk factors for coronary artery disease and myocardial infarction," Journal of the American College of Cardiology, vol. 32, no. 1, pp. 35-41, 1998.

[45] H. Toss, J. Gnarpe, H. Gnarpe, A. Siegbahn, B. Lindahl, and L. Wallentin, "Increased fibrinogen levels are associated with persistent Chlamydia pneumoniae infection in unstable coronary artery disease," European Heart Journal, vol. 19, no. 4, pp. 570-577, 1998.

[46] M. Gattone, L. Iacoviello, M. Colombo et al., "Chlamydia pneumoniae and cytomegalovirus seropositivity, inflammatory markers, and the risk of myocardial infarction at a young age," American Heart Journal, vol. 142, no. 4, pp. 633-640, 2001.

[47] D. Sander, K. Winbeck, J. Klingelhöfer, T. Etgen, and B. Conrad, "Enhanced progression of early carotid atherosclerosis is related to Chlamydia pneumoniae (Taiwan acute respiratory) seropositivity," Circulation, vol. 103, no. 10, pp. 1390-1395, 2001.
[48] R. Altman, J. Rouvier, A. Scazziota, and C. Gonzalez, "No causal association between inflammation and Chlamydia pneumoniae in patients with chronic ischemic arterial disease," Inflammation, vol. 26, no. 1, pp. 25-30, 2002.

[49] D. G. M. Bloemenkamp, W. P. T. M. Mali, B. C. Tanis et al., "Chlamydia pneumoniae, Helicobacter pylori and cytomegalovirus infections and the risk of peripheral arterial disease in young women," Atherosclerosis, vol. 163, no. 1, pp. 149-156, 2002.

[50] A. Schumacher, I. Seljeflot, A. B. Lerkerod, L. Sommervoll, J. E. Otterstad, and H. Arnesen, "Positive Chlamydia pneumoniae serology is associated with elevated levels of tumor necrosis factor alpha in patients with coronary heart disease," Atherosclerosis, vol. 164, no. 1, pp. 153-160, 2002.

[51] L. M. Biasucci, G. Liuzzo, A. Ciervo et al., "Antibody response to chlamydial heat shock protein 60 is strongly associated with acute coronary syndromes," Circulation, vol. 107, no. 24, pp. 3015-3017, 2003.

[52] A. S. Gabriel, S. Ahnve, H. Gnarpe, J. Gnarpe, and A. Martinsson, "Azithromycin therapy in patients with chronic Chlamydia pneumoniae infection and coronary heart disease: immediate and long-term effects on inflammation, coagulation, and lipid status in a double-blind, placebo-controlled study," European Journal of Internal Medicine, vol. 14, no. 8, pp. 470-478, 2003.

[53] J.-L. Georges, H. J. Rupprecht, S. Blankenberg et al., "Impact of pathogen burden in patients with coronary artery disease in relation to systemic inflammation and variation in genes encoding cytokines," The American Journal of Cardiology, vol. 92, no. 5, pp. 515-521, 2003.

[54] M. N. Zairis, O. A. Papadaki, P. K. Psarogianni et al., "Serologic markers of persistent Chlamydia pneumonia infection and longterm prognosis after successful coronary stenting," American Heart Journal, vol. 146, no. 6, pp. 1082-1089, 2003.

[55] G. A. Lanza, A. Sestito, G. Cammarota et al., "Assessment of systemic inflammation and infective pathogen burden in patients with cardiac syndrome X," American Journal of Cardiology, vol. 94, no. 1, pp. 40-44, 2004.

[56] J. P. Linares-Palomino, J. Gutiérrez, C. Lopez-Espada, J. D. D. Luna, E. Ros, and C. Maroto, "Genomic, serologic, and clinical case-control study of Chlamydia pneumoniae and peripheral artery occlusive disease," Journal of Vascular Surgery, vol. 40, no. 2, pp. 359-366, 2004.

[57] A. K. Adiloglu, R. Can, C. Nazli et al., "Ectasia and severe atherosclerosis: relationships with Chlamydia pneumoniae, Helicobacter pylori, and inflammatory markers," Texas Heart Institute Journal, vol. 32, no. 1, pp. 21-27, 2005.

[58] A. Schumacher, I. Seljeflot, A. B. Lerkerød, L. Sommervoll, J. E. Otterstad, and H. Arnesen, "Chlamydia LPS and MOMP seropositivity are associated with different cytokine profiles in patients with coronary heart disease," European Journal of Clinical Investigation, vol. 35, no. 7, pp. 431-437, 2005.

[59] E. Corrado, M. Rizzo, R. Tantillo et al., "Markers of inflammation and infection influence the outcome of patients with baseline asymptomatic carotid lesions: a 5-year follow-up study," Stroke, vol. 37, no. 2, pp. 482-486, 2006.

[60] K. Jitsuiki, K. Yamane, M. Nakajima et al., "Association of Chlamydia pneumoniae infection and carotid intima-media wall thickness in Japanese Americans," Circulation Journal, vol. 70, no. 7, pp. 815-819, 2006.

[61] E. A. Kaperonis, C. D. Liapis, J. D. Kakisis et al., "Inflammation and Chlamydia pneumoniae infection correlate with the severity 
of peripheral arterial disease," European Journal of Vascular and Endovascular Surgery, vol. 31, no. 5, pp. 509-515, 2006.

[62] C. Romano Carratelli, I. Nuzzo, D. Cozzolino, C. Bentivoglio, R. Paolillo, and A. Rizzo, "Relationship between Chlamydia pneumoniae infection, inflammatory markers, and coronary heart diseases," International Immunopharmacology, vol. 6, no. 5, pp. 848-853, 2006.

[63] V. Videm, R. Wiseth, S. Gunnes, H. O. Madsen, and P. Garred, "Multiple inflammatory markers in patients with significant coronary artery disease," International Journal of Cardiology, vol. 118, no. 1, pp. 81-87, 2007.

[64] A. Jafarzadeh, A. Esmaeeli-Nadimi, and M. Shariati, "High sensitivity C-reactive protein and immunoglobulin $\mathrm{G}$ against Chlamydia Pneumoniae and Chlamydial heat shock protein-60 in ischemic heart disease," Iranian Journal of Immunology, vol. 5, no. 1, pp. 51-56, 2008.

[65] H. C. Jha, P. Srivastava, R. Sarkar, J. Prasad, and A. S. Mittal, "Association of plasma circulatory markers, Chlamydia pneumoniae, and high sensitive C-reactive protein in coronary artery disease patients of India," Mediators of Inflammation, vol. 2009, Article ID 561532, 5 pages, 2009.

[66] A. Nazmi, A. V. Diez-Roux, N. S. Jenny, M. Y. Tsai, M. Szklo, and A. E. Aiello, "The influence of persistent pathogens on circulating levels of inflammatory markers: a cross-sectional analysis from the Multi-Ethnic Study of Atherosclerosis," BMC Public Health, vol. 10, article 706, 2010.

[67] M. Haider, M. Rizvi, A. Malik, M. Azam, and M. U. Rabbani, "Acute and chronic Chlamydia pneumoniae infection and inflammatory markers in coronary artery disease patients," Journal of Infection in Developing Countries, vol. 5, no. 8, pp. 580-586, 2011.

[68] L. A. Mundkur, V. S. Rao, S. Hebbagudi et al., "Pathogen burden, cytomegalovirus infection and inflammatory markers in the risk of premature coronary artery disease in individuals of Indian origin," Experimental and Clinical Cardiology, vol. 17, no. 2, pp. 63-68, 2012.

[69] A. K. Pandey, S. Pandey, M. J. Blaha et al., "Family history of coronary heart disease and markers of subclinical cardiovascular disease: where do we stand?" Atherosclerosis, vol. 228, no. 2, pp. 285-294, 2013. 


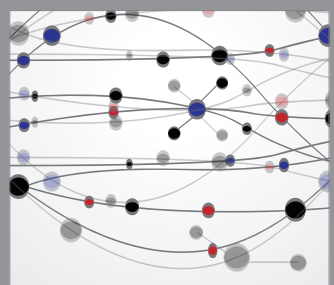

The Scientific World Journal
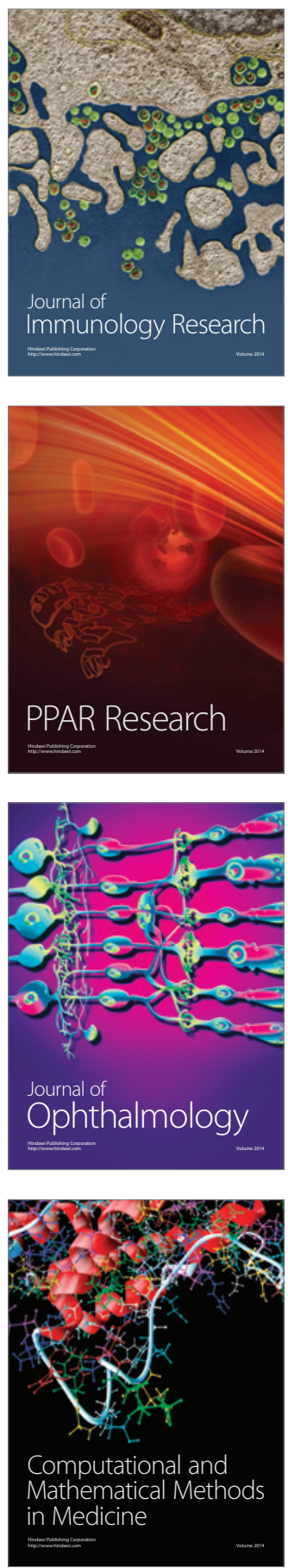

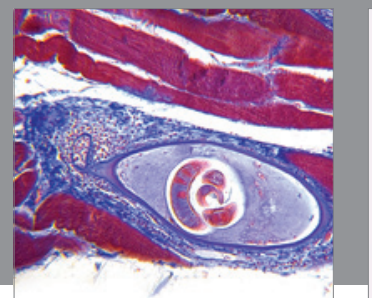

Gastroenterology

Research and Practice
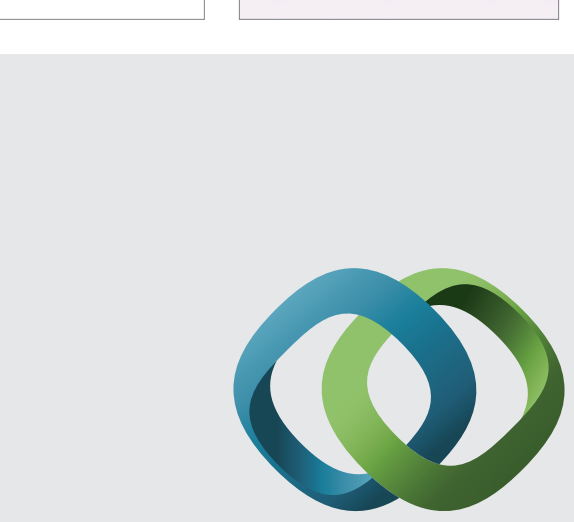

\section{Hindawi}

Submit your manuscripts at

http://www.hindawi.com
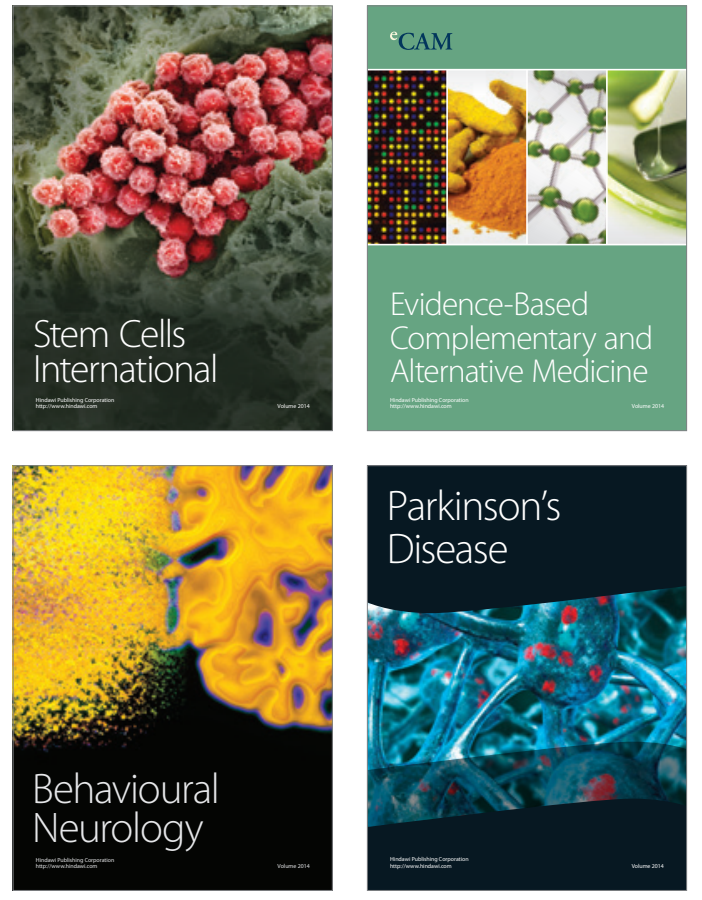
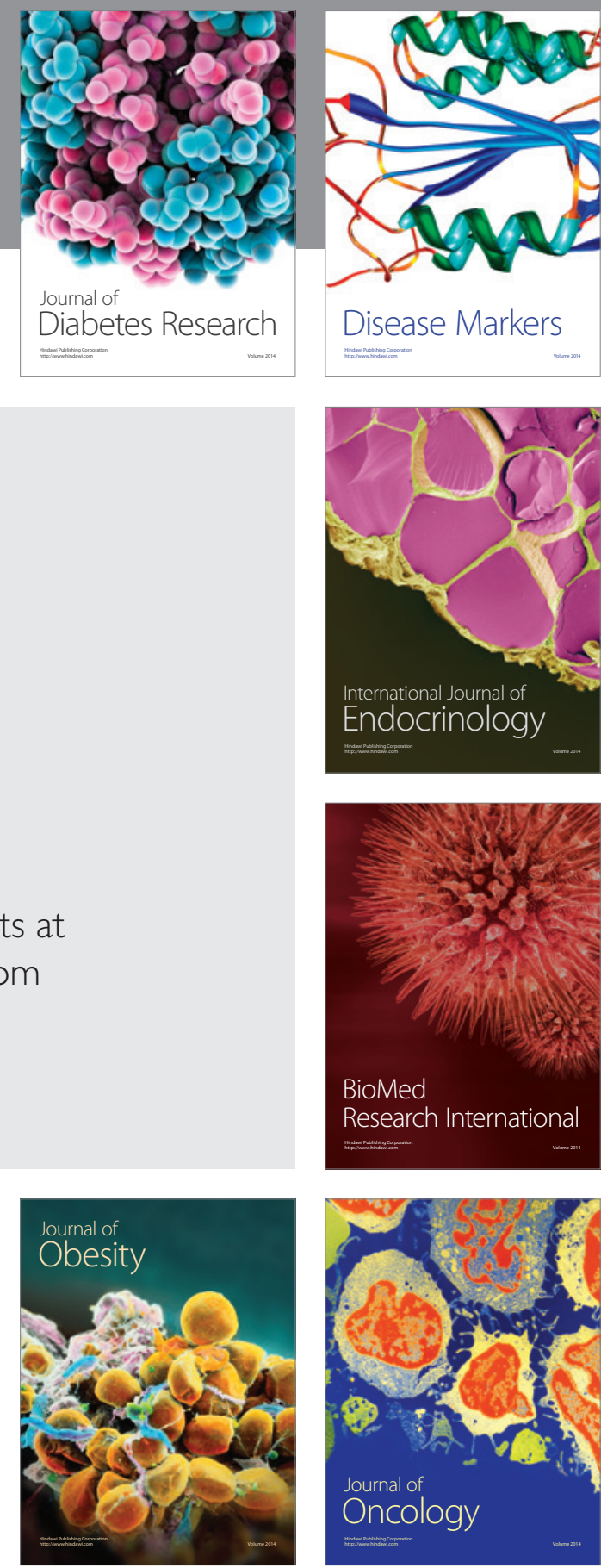

Disease Markers
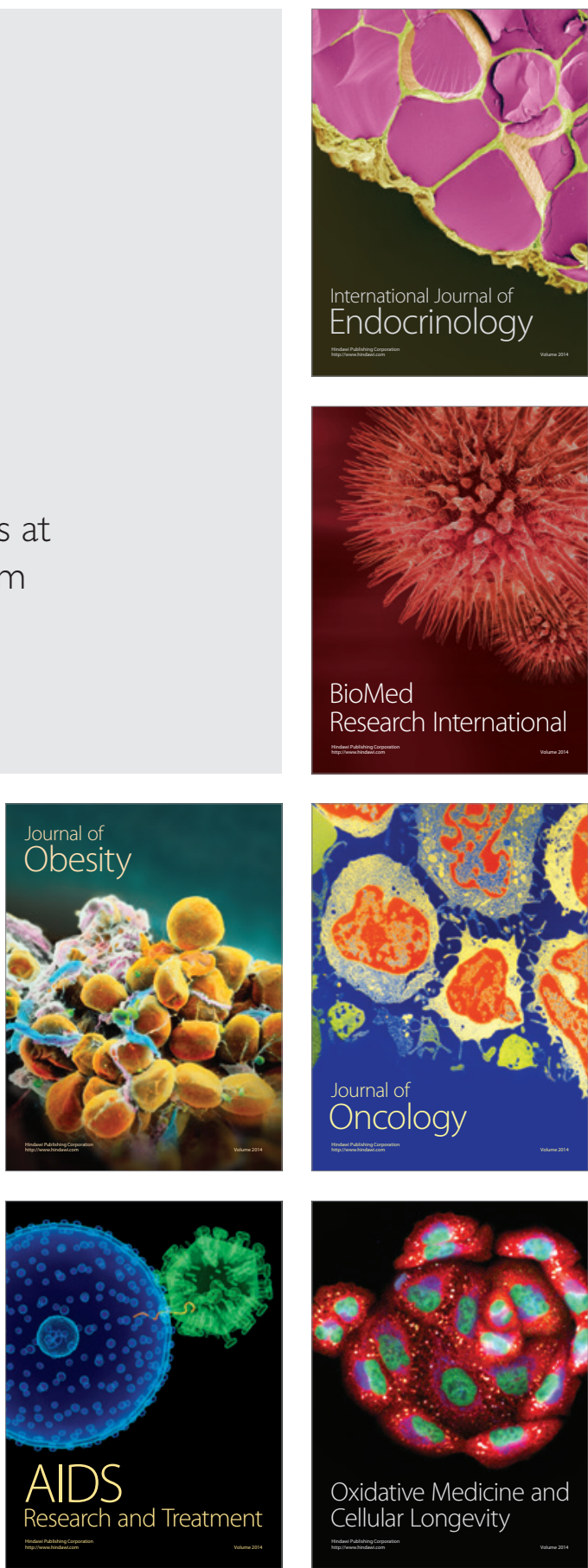